\title{
A NOTE ON THE SECOND MOMENT OF AUTOMORPHIC $L$-FUNCTIONS
}

\author{
H. M. BUI
}

\begin{abstract}
We obtain the formula for the twisted harmonic second moment of the $L$-functions associated with primitive Hecke eigenforms of weight 2. A consequence of our mean value theorem is reminiscent of recent results of Conrey and Young on the reciprocity formula for the twisted second moment of Dirichlet $L$-functions.
\end{abstract}

\section{INTRODUCTION}

In this paper, we study the twisted second moment of the family of $L$-functions arising from $\mathcal{S}_{2}^{*}(q)$, the set of primitive Hecke eigenforms of weight 2 , lever $q$ ( $q$ prime). For $f(z) \in \mathcal{S}_{2}^{*}(q), f$ has a Fourier expansion

$$
f(z)=\sum_{n=1}^{\infty} n^{1 / 2} \lambda_{f}(n) e(n z)
$$

where the normalization is such that $\lambda_{f}(1)=1$. The $L$-function associated to $f$ has an Euler product

$$
L(f, s)=\sum_{n=1}^{\infty} \frac{\lambda_{f}(n)}{n^{s}}=\left(1-\frac{\lambda_{f}(q)}{q^{s}}\right)^{-1} \prod_{h}\left(1-\frac{\lambda_{f}(h)}{h^{s}}+\frac{1}{h^{2 s}}\right)^{-1} .
$$

The series is absolutely convergent when $\Re s>1$, and admits analytic continuation to all of $\mathbb{C}$. The functional equation for $L(f, s)$ is

$$
\Lambda(f, s):=\left(\frac{\sqrt{q}}{2 \pi}\right)^{s} \Gamma\left(s+\frac{1}{2}\right) L(f, s)=\varepsilon_{f} \Lambda(f, 1-s)
$$

where $\varepsilon_{f}=-q^{1 / 2} \lambda_{f}(q)= \pm 1$. We define the harmonic average as

$$
\sum_{f}^{h} A_{f}:=\sum_{f \in \mathcal{S}_{2}^{*}(q)} \frac{A_{f}}{4 \pi(f, f)},
$$

where $(f, g)$ is the Petersson inner product on the space $\Gamma_{0}(q) \backslash \mathbb{H}$.

We are interested in the twisted second moment of this family of $L$-functions. We define

$$
S(p, q)=\sum_{f \in \mathcal{S}_{2}^{*}(q)}^{h} L\left(f, \frac{1}{2}\right)^{2} \lambda_{f}(p) .
$$

Our main theorem is

Theorem 1. Suppose $q$ is prime and $0<p \leq C q$, for some fixed $C<1$. Then we have

$$
S(p, q)=\frac{d(p)}{\sqrt{p}} \log \frac{q}{4 \pi^{2} p}+O\left(p^{1 / 2} q^{-1+\varepsilon}\right) .
$$


Remark 1. The twisted harmonic fourth moment has been considered by Kowalski, Michel and VanderKam [6], where they gave an asymptotic formula for the fourth power mean value provided that $p \ll q^{1 / 9-\varepsilon}$.

Remark 2. In a similar setting, Iwaniec and Sarnak 3] have given the exact formula for the twisted second moment of the automorphic $L$-functions arising from $\mathcal{H}_{k}(1)$, the set of newforms in $\mathcal{S}_{k}(1)$, where $\mathcal{S}_{k}(1)$ is the linear space of holomorphic cusp forms of weight $k$. Precisely, they showed that for $k>2, k \equiv 0(\bmod 2)$, and for any $m \geq 1$, we have

$$
\begin{array}{r}
\frac{12}{k-1} \sum_{f \in \mathcal{H}_{k}(1)} w_{f} L\left(f, \frac{1}{2}\right)^{2} \lambda_{f}(m)=2\left(1+i^{k}\right) \frac{d(m)}{\sqrt{m}}\left(\sum_{0<l \leq k / 2} \frac{1}{l}-\log 2 \pi \sqrt{m}\right) \\
-\frac{2 \pi i^{k}}{\sqrt{m}} \sum_{h \neq m} d(h) d(h-m) p_{k}\left(\frac{h}{m}\right)+\frac{2 \pi i^{k}}{\sqrt{m}} \sum_{h} d(h) d(h+m) q_{k}\left(\frac{h}{m}\right),
\end{array}
$$

where $p_{k}(x)$ and $q_{k}(x)$ are Hankel transforms of Bessel functions

$$
p_{k}(x)=\int_{0}^{\infty} Y_{0}(y \sqrt{x}) J_{k-1}(y) d y, \text { and } q_{k}(x)=\frac{2}{\pi} \int_{0}^{\infty} K_{0}(y \sqrt{x}) J_{k-1}(y) d y .
$$

Here the weight $w_{f}=\zeta(2) L\left(\operatorname{sym}^{2}(f), 1\right)^{-1}$, where the symmetric square $L$-function $L\left(\operatorname{sym}^{2}(f), s\right)$ corresponding to $f$ is defined by

$$
L\left(\operatorname{sym}^{2}(f), s\right)=\zeta(2 s) \sum_{n=1}^{\infty} \frac{\lambda_{f}\left(n^{2}\right)}{n^{s}} .
$$

In the context of Dirichlet $L$-functions, consider

$$
M(p, q)=\frac{1}{\varphi^{*}(q)} \sum_{\chi(\bmod q)}^{*}\left|L\left(\frac{1}{2}, \chi\right)\right|^{2} \chi(p),
$$

where $\sum^{*}$ denotes summation over all primitive characters $\chi(\bmod q)$, and $\varphi^{*}(q)$ is the number of primitive characters. This is the twisted second moment of Dirichlet $L$ functions. In a recent paper, Conrey [1] proved that there is a kind of reciprocity formula relating $M(p, q)$ and $M(-q, p)$ when $p$ and $q$ are distinct prime integers. Precisely, Conrey showed that

$$
M(p, q)=\frac{\sqrt{p}}{\sqrt{q}} M(-q, p)+\frac{1}{\sqrt{p}}\left(\log \frac{q}{p}+A\right)+\frac{B}{2 \sqrt{q}}+O\left(\frac{p}{q}+\frac{\log q}{q}+\frac{\log p q}{\sqrt{p q}}\right),
$$

where $A$ and $B$ are some explicit constants. This provides an asymptotic formula for $M(p, q)-\sqrt{p / q} M(-q, p)$ under the condition that $p \ll q^{2 / 3-\varepsilon}$. The error term above was improved by Young [7] so that the asymptotic formula holds for $p \ll q^{1-\varepsilon}$.

We now take $p$ to be prime and, similarly as before, $S(q, p)$ is defined as the harmonic second moment, twisted by $\lambda_{g}(q)$, of the family of $L$-functions arising from $g(z) \in \mathcal{S}_{2}^{*}(p)$. We note that as $q$ is prime, the Ramanujan bound $\left|\lambda_{f}(n)\right| \leq d(n)[2$ yields

$$
S(q, p) \ll \sum_{g \in \mathcal{S}_{2}^{*}(p)}^{h} L\left(g, \frac{1}{2}\right)^{2} \ll \log p .
$$

Thus as a trivial consequence of Theorem 1 , for $p<q$ we have

$$
S(p, q)-\sqrt{p / q} S(q, p)=\frac{2}{\sqrt{p}} \log \frac{q}{4 \pi^{2} p}+O\left(p^{1 / 2+\varepsilon} q^{-1 / 2}\right) .
$$


This leads to an asymptotic formula for $S(p, q)-\sqrt{p / q} S(q, p)$, at least for $p$ as large as $q^{1 / 2-\varepsilon}$. The results in the Dirichlet $L$-functions case [1]7] suggest that the asymptotic formula should hold for $p \ll q^{\theta}$, for any $\theta<1$. However, our technique fails to extend the range to any power $\theta>1 / 2$. For that purpose, we need more refined estimates for the off-diagonal terms of $S(p, q)$ and $S(q, p)$. The intricate calculations seem to suggest that there is a large cancellation between these two expressions. The nature of this is not well-understood.

\section{Preliminary Lemmas}

We require some lemmas. We begin with Hecke's formula for primitive forms.

Lemma 1. For $m, n \geq 1$,

$$
\lambda_{f}(m) \lambda_{f}(n)=\sum_{\substack{d \mid(m, n) \\(d, q)=1}} \lambda_{f}\left(\frac{m n}{d^{2}}\right) .
$$

The next lemma is a particular case of Petersson's trace formula.

Lemma 2. For $m, n \geq 1$, we have

$$
\sum_{f \in \mathcal{S}_{2}^{*}(q)}^{h} \lambda_{f}(m) \lambda_{f}(n)=\delta_{m, n}-J_{q}(m, n)
$$

where $\delta_{m, n}$ is the Kronecker symbol and

$$
J_{q}(m, n)=2 \pi \sum_{c=1}^{\infty} \frac{S(m, n ; c q)}{c q} J_{1}\left(\frac{4 \pi \sqrt{m n}}{c q}\right) .
$$

Here $J_{1}(x)$ is the Bessel function of order 1 , and $S(m, n ; c)$ is the Kloosterman sum

$$
S(m, n ; c)=\sum_{a(\bmod c)}^{*} e\left(\frac{m a+n \bar{a}}{c}\right) .
$$

Moreover we have

$$
J_{q}(m, n) \ll(m, n, q)^{1 / 2}(m n)^{1 / 2+\varepsilon} q^{-3 / 2} .
$$

The above estimate follows easily from the bound $J_{1}(x) \ll x$ and Weil's bound on Kloosterman sums.

We mention a result of Jutila [4 (cf. Theorem 1.7), which is an extension of the Voronoi summation formula.

Lemma 3. Let $f: \mathbb{R}^{+} \rightarrow \mathbb{C}$ be a $C^{\infty}$ function which vanishes in the neighbourhood of 0 and is rapidly decreasing at infinity. Then for $c \geq 1$ and $(a, c)=1$,

$$
\begin{aligned}
c \sum_{m=1}^{\infty} d(m) e\left(\frac{a m}{c}\right) f(m)= & 2 \int_{0}^{\infty}\left(\log \frac{\sqrt{x}}{c}+\gamma\right) f(x) d x \\
& -2 \pi \sum_{m=1}^{\infty} d(m) e\left(\frac{-\bar{a} m}{c}\right) \int_{0}^{\infty} Y_{0}\left(\frac{4 \pi \sqrt{m x}}{c}\right) f(x) d x \\
& +4 \sum_{m=1}^{\infty} d(m) e\left(\frac{\bar{a} m}{c}\right) \int_{0}^{\infty} K_{0}\left(\frac{4 \pi \sqrt{m x}}{c}\right) f(x) d x .
\end{aligned}
$$


The next lemma concerns the approximate functional equation for $L$-functions.

Lemma 4. Let $G(s)$ be an even entire function satisfying $G(0)=1$ and $G$ has a double zero at each $s \in \mathbb{Z}$. Furthermore let assume that $G(s) \ll_{A, B}(1+|s|)^{-A}$ for any $A>0$ in any strip $-B \leq \Re s \leq B$. Then for $f \in \mathcal{S}_{2}^{*}(q)$,

$$
L\left(f, \frac{1}{2}\right)^{2}=2 \sum_{n=1}^{\infty} \frac{d(n) \lambda_{f}(n)}{\sqrt{n}} W_{q}\left(\frac{4 \pi^{2} n}{q}\right),
$$

where

$$
W_{q}(x)=\frac{1}{2 \pi i} \int_{(1)} G(s) \Gamma(s+1)^{2} \zeta_{q}(2 s+1) x^{-s} \frac{d s}{s} .
$$

Here $\zeta_{q}(s)$ is defined by

$$
\zeta_{q}(s)=\sum_{\substack{n=1 \\ n, q)=1}}^{\infty} n^{-s} \quad(\sigma>1) .
$$

Proof. From Lemma 1 we first note that

$$
L(f, s)^{2}=\zeta_{q}(2 s) \sum_{n=1}^{\infty} \frac{d(n) \lambda_{f}(n)}{n^{s}} \quad(\sigma>1) .
$$

Consider

$$
A(f):=\frac{1}{2 \pi i} \int_{(1)} \frac{G(s) \Lambda\left(f, s+\frac{1}{2}\right)^{2}}{\frac{\sqrt{q}}{2 \pi}} \frac{d s}{s} .
$$

Moving the line of integration to $\Re s=-1$, and applying Cauchy's theorem and the functional equation, we derive that $A(f)=L\left(f, \frac{1}{2}\right)^{2}-A(f)$. Expanding $\Lambda\left(f, s+\frac{1}{2}\right)^{2}$ in a Dirichlet series and integrating termwise we obtain the lemma.

For our purpose, $W_{q}$ is basically a "cut-off" function. Indeed, we have the following.

Lemma 5. The function $W_{q}$ satisfies

$$
\begin{gathered}
W_{q}^{(j)}(x) \ll_{j, N} x^{-N} \text { for } x \geq 1 \text { and all } j, N \geq 0, \\
x^{i} W_{q}^{(j)}(x) \ll_{i, j}|\log x| \text { for } 0<x<1 \text { and all } i \geq j \geq 0,
\end{gathered}
$$

and

$$
W_{q}(x)=-\left(1-\frac{1}{q}\right) \frac{\log x}{2}+\frac{\log q}{q}+O_{N}\left(x^{N}\right) \text { for } 0<x<1 \text { and all } N \geq 0 .
$$

The implicit constants are independent of $q$.

Proof. The first estimate is a direct consequence of Stirling's formula after differentiating under the integral sign and shifting the line of integration to $\Re s=N$. The only difference in the other two estimates is that one has to move the line of integration to $\Re s=-N$. 


\section{Proof of Theorem 1}

Our argument in this section follows closely [5]. From Lemma 4 and Lemma 2 we obtain

$$
S(p, q)=2 \frac{d(p)}{\sqrt{p}} W_{q}\left(\frac{4 \pi^{2} p}{q}\right)-2 R(p, q)
$$

where

$$
R(p, q)=\sum_{n=1}^{\infty} \frac{d(n)}{\sqrt{n}} J_{q}(n, p) W_{q}\left(\frac{4 \pi^{2} n}{q}\right) .
$$

Using Lemma 5, the first term is

$$
\frac{d(p)}{\sqrt{p}} \log \frac{q}{4 \pi^{2} p}+O\left(p^{-1 / 2} q^{-1+\varepsilon}+p^{1 / 2+\varepsilon} q^{-1}\right) .
$$

Thus, we are left to consider $R(p, q)$. We have

$$
R(p, q)=2 \pi \sum_{n=1}^{\infty} \frac{d(n)}{\sqrt{n}} \sum_{c=1}^{\infty} \frac{S(n, p ; c q)}{c q} J_{1}\left(\frac{4 \pi \sqrt{n p}}{c q}\right) W_{q}\left(\frac{4 \pi^{2} n}{q}\right) .
$$

Using Weil's bound for Kloosterman sums and $J_{1}(x) \ll x$, the contribution from the terms $c \geq q$ is

$$
\ll p^{1 / 2} q^{-3 / 2} \sum_{n=1}^{\infty}(n, p)^{1 / 2} d(n)\left|W_{q}\left(\frac{4 \pi^{2} n}{q}\right)\right| \sum_{c \geq q} \frac{d(c)}{c^{3 / 2}} \ll p^{1 / 2} q^{-1+\varepsilon} .
$$

Thus we need to study

$$
\frac{2 \pi}{q} \sum_{c<q} \frac{1}{c} \sum_{a(\bmod c q)}^{*} e\left(\frac{\bar{a} p}{c q}\right) \sum_{n=1}^{\infty} d(n) e\left(\frac{a n}{c q}\right) \frac{J_{1}\left(\frac{4 \pi \sqrt{n p}}{c q}\right) W_{q}\left(\frac{4 \pi^{2} n}{q}\right)}{\sqrt{n}} .
$$

We fix a $C^{\infty}$ function $\xi: \mathbb{R}^{+} \rightarrow[0,1]$, which satisfies $\xi(x)=0$ for $0 \leq x \leq 1 / 2$ and $\xi(x)=1$ for $x \geq 1$, and attach the weight $\xi(n)$ to the innermost sum. Using Lemma 3 , this is equal to

$$
\frac{4 \pi}{q^{2}} \sum_{c<q} \frac{1}{c^{2}} S(0, p ; c q) \int_{0}^{\infty}\left(\log \frac{\sqrt{t}}{c q}+\gamma\right) J_{1}\left(\frac{4 \pi \sqrt{t p}}{c q}\right) W_{q}\left(\frac{4 \pi^{2} t}{q}\right) \xi(t) \frac{d t}{\sqrt{t}}-Y+K
$$

where

$$
\begin{aligned}
Y=\frac{4 \pi^{2}}{q^{2}} \sum_{c<q} & \frac{1}{c^{2}} \sum_{n=1}^{\infty} d(n) S(0, p-n ; c q) \\
& \int_{0}^{\infty} Y_{0}\left(\frac{4 \pi \sqrt{n t}}{c q}\right) J_{1}\left(\frac{4 \pi \sqrt{t p}}{c q}\right) W_{q}\left(\frac{4 \pi^{2} t}{q}\right) \xi(t) \frac{d t}{\sqrt{t}}
\end{aligned}
$$

and

$$
\begin{aligned}
K=\frac{8 \pi}{q^{2}} \sum_{c<q} & \frac{1}{c^{2}} \sum_{n=1}^{\infty} d(n) S(0, p+n ; c q) \\
& \int_{0}^{\infty} K_{0}\left(\frac{4 \pi \sqrt{n t}}{c q}\right) J_{1}\left(\frac{4 \pi \sqrt{t p}}{c q}\right) W_{q}\left(\frac{4 \pi^{2} t}{q}\right) \xi(t) \frac{d t}{\sqrt{t}} .
\end{aligned}
$$


We will deal with $Y$ and $K$ in the next three lemmas. For the first sum, since $S(0, p ; c q)=\mu(q) S(0, p \bar{q} ; c)$ and $J_{1}(x) \ll x$, this is

$$
\ll p^{1 / 2} q^{-3} \sum_{c<q} \frac{1}{c^{2}} \int_{1 / 2}^{\infty}\left|W_{q}\left(\frac{4 \pi^{2} t}{q}\right)\right|(\log t c q) d t \ll p^{1 / 2} q^{-2+\varepsilon} .
$$

Lemma 6. For $K$ defined as in (2), we have

$$
K \ll p^{1 / 2} q^{\varepsilon}(q-p)^{-1+\varepsilon} \text {. }
$$

And hence $K \ll p^{1 / 2} q^{-1+\varepsilon}$, given that $p \leq C q$ for some fixed $C<1$.

Remark 3. This is the only place where the condition $p \leq C q$ for some constant $C<1$ is used.

Proof. The integral involving $K_{0}$, using $K_{0}(y) \ll y^{-1 / 2} e^{-y}$, is

$$
\begin{aligned}
& \int_{0}^{\infty} K_{0}\left(\frac{4 \pi \sqrt{n t}}{c q}\right) J_{1}\left(\frac{4 \pi \sqrt{t p}}{c q}\right) W_{q}\left(\frac{4 \pi^{2} t}{q}\right) \xi(t) \frac{d t}{\sqrt{t}} \\
= & \frac{c q}{2 \pi \sqrt{n}} \int_{0}^{\infty} K_{0}(y) J_{1}\left(\sqrt{\frac{p}{n}} y\right) W_{q}\left(\frac{c^{2} q y^{2}}{4 n}\right) \xi\left(\frac{c^{2} q^{2} y^{2}}{16 \pi^{2} n}\right) d y \\
\ll & \frac{c p^{1 / 2} q^{1+\varepsilon}}{n} \int_{\sqrt{n} / c q}^{\infty} y^{1 / 2} e^{-y} d y \ll \frac{c p^{1 / 2} q^{1+\varepsilon}}{n} e^{-\sqrt{n} / 2 c q} .
\end{aligned}
$$

Thus, as $S(0, p+n ; c q)=S(0,(p+n) \bar{q} ; c) S(0, p+n ; q)$ and $|S(0,(p+n) \bar{q} ; c)| \leq \sum_{l \mid(p+n, c)} l$,

$$
\begin{aligned}
K & \ll p^{1 / 2} q^{-1+\varepsilon} \sum_{n=1}^{\infty} \frac{d(n)}{n} e^{-\sqrt{n} / 2 q^{2}}|S(0, p+n ; q)| \sum_{c<q} \frac{\sum_{l \mid(p+n, c)} l}{c} \\
& \ll p^{1 / 2} q^{-1+\varepsilon} \sum_{n=1}^{\infty} \frac{d(n) d(p+n)}{n} e^{-\sqrt{n} / 2 q^{2}}|S(0, p+n ; q)| .
\end{aligned}
$$

We break the sum over $n$ according to whether $q \mid(p+n)$ or $q \nmid(p+n)$. The contribution of the latter is $O\left(p^{1 / 2} q^{-1+\varepsilon}\right)$. That of the former is

$$
\ll p^{1 / 2} q^{\varepsilon} \sum_{l=1}^{\infty} \frac{d(l) d(q l-p)}{q l-p} e^{-\sqrt{q l-p} / 2 q^{2}} \ll p^{1 / 2} q^{\varepsilon}(q-p)^{-1+\varepsilon}+p^{1 / 2} q^{-1+\varepsilon} .
$$

The lemma follows.

The case of $Y$ is more complicated as $Y_{0}$ is an oscillating function. For that we need the following standard lemma (for example, see [5]).

Lemma 7. Let $v \geq 0$ and $J$ be a positive integer. If $f$ is a compactly supported $C^{\infty}$ function on $[Y, 2 Y]$, and there exists $\beta>0$ such that

$$
y^{j} f^{(j)}(y) \ll_{j}(1+\beta Y)^{j}
$$

for $0 \leq j \leq J$, then for any $\alpha>1$, we have

$$
\int_{0}^{\infty} Y_{v}(\alpha y) f(y) d y \ll\left(\frac{1+\beta Y}{1+\alpha Y}\right)^{J} Y .
$$

Lemma 8. For $Y$ defined as in (1), we have

$$
Y \ll p^{1 / 2} q^{-1+\varepsilon} .
$$


Proof. We have

$$
Y=\frac{4 \pi^{2}}{q^{2}} \sum_{c<q} \frac{1}{c^{2}} \sum_{n=1}^{\infty} d(n) S(0, p-n ; c q) y(n),
$$

where

$$
y(n)=\int_{0}^{\infty} Y_{0}\left(\frac{4 \pi \sqrt{n t}}{c q}\right) J_{1}\left(\frac{4 \pi \sqrt{t p}}{c q}\right) W_{q}\left(\frac{4 \pi^{2} t}{q}\right) \xi(t) \frac{d t}{\sqrt{t}} .
$$

We make a smooth dyadic partition of unity that $\xi=\sum_{k} \xi_{k}$, where each $\xi_{k}$ is a compactly supported $C^{\infty}$ function on the dyadic interval $\left[X_{k}, 2 X_{k}\right]$. Moreover, $\xi_{k}$ satisfies $x^{j} \xi_{k}^{(j)}(x) \ll 1$, for all $j \geq 0$. We work on each $\xi_{k}$ individually, but we write $\xi$ instead of $\xi_{k}$ and, accordingly, $X$ rather than $X_{k}$.

By the change of variable $x:=2 \sqrt{t} / c q$, we have

$$
y(n)=c q \int_{0}^{\infty} Y_{0}(2 \pi \sqrt{n} x) J_{1}(2 \pi \sqrt{p} x) W_{q}\left(\pi^{2} c^{2} q x^{2}\right) \xi\left(\frac{c^{2} q^{2} x^{2}}{4}\right) d x
$$

We define

$$
f(x):=J_{1}(2 \pi \sqrt{p} x) W_{q}\left(\pi^{2} c^{2} q x^{2}\right) \xi\left(\frac{c^{2} q^{2} x^{2}}{4}\right) .
$$

This is a $C^{\infty}$ function compactly supported on $[\rho, 2 \rho]$, where $\rho=2 \sqrt{X} / c q$.

We first treat the case $1 / 2 \leq X \leq q$. We note that this involves $O(\log q)$ dyadic intervals. From Lemma 5 we have $x^{j} W^{(j)}(x) \ll_{j} \log q$ for $1 / q \ll x \ll 1$. This, together with the recurrence relation $\left(x^{v} J_{v}(x)\right)^{\prime}=x^{v} J_{v-1}(x)$, gives

$$
x^{j} f^{(j)}(x) \ll_{j}(1+\sqrt{p} x)^{j} \log q .
$$

We are in a position to apply Lemma 7 to $f$ with $\alpha=2 \pi \sqrt{n}, \beta=\sqrt{p}$ and $Y=\rho=$ $2 \sqrt{X} / c q$. The lemma yields, for any positive integer $J$,

$$
y(n) \ll c q \rho\left(\frac{1+\sqrt{p} \rho}{1+\sqrt{n} \rho}\right)^{J} \log q .
$$

Later, we will break the sum over $n$ in (3) in the following way

$$
\sum_{n \geq 1}=\sum_{n \leq \rho^{-\kappa}}+\sum_{n>\rho^{-\kappa}}
$$

where $\kappa>2$ will be chosen later. The estimate ([6) will be used for $n>\rho^{-\kappa}$. We need another estimate for the range $n \leq \rho^{-\kappa}$. For this we go back to (4), using $Y_{0}(x) \ll$ $1+|\log x|$ and $J_{1}(x) \ll x$, to derive

$$
y(n) \ll \frac{\sqrt{p} X}{c q}(\log q)^{2} .
$$


We denote by $Y_{1}$ and $Y_{2}$ the corresponding splitted sums $\left(Y=Y_{1}+Y_{2}\right)$. For the first sum, using (7), we have

$$
\begin{aligned}
Y_{1} & =\frac{4 \pi^{2}}{q^{2}} \sum_{c<q} \frac{1}{c^{2}} \sum_{n \leq \rho^{-\kappa}} d(n) S(0, p-n ; c q) y(n) \\
& \ll p^{1 / 2} X q^{-3+\varepsilon} \sum_{n \leq \rho^{-\kappa}} d(n)|S(0, p-n ; q)| \sum_{c<q} \frac{1}{c^{3}} \sum_{l \mid(p-n, c)} l \\
& \ll p^{1 / 2} X q^{-3+\varepsilon} \sum_{n \leq\left(\frac{q^{2}}{2 \sqrt{X}}\right)^{\kappa}} d(n)|S(0, p-n ; q)| \sum_{\frac{2 \sqrt{X}}{q} n^{1 / \kappa} \leq c<q} \sum_{l \mid(p-n, c)} \frac{l}{c^{3}} \\
& \ll p^{1 / 2} q^{-1+\varepsilon} \sum_{n \leq\left(\frac{q^{2}}{2 \sqrt{X}}\right)^{\kappa}} \frac{d(n) d(p-n)}{n^{2 / \kappa}}|S(0, p-n ; q)| \\
& \ll p^{1 / 2} q^{2 \kappa-5+\varepsilon} .
\end{aligned}
$$

For the second sum, we note that $\sqrt{p} \rho \ll 1$ in this range. Using (6), we have

$$
y(n) \ll \sqrt{X}(\log q) n^{-J / 2} \rho^{-J} .
$$

Similarly to above, we deduce that

$$
\begin{aligned}
Y_{2} & =\frac{4 \pi^{2}}{q^{2}} \sum_{c<q} \frac{1}{c^{2}} \sum_{n>\rho^{-\kappa}} d(n) S(0, p-n ; c q) y(n) \\
& \ll \sqrt{X} q^{-2+\varepsilon} \sum_{n>\rho^{-\kappa}} \frac{d(n)}{n^{J / 2}}|S(0, p-n ; q)| \sum_{c<q} \frac{1}{c^{2}} \sum_{l \mid(p-n, c)} l \rho^{-J} \\
& \ll X^{-(J-1) / 2} q^{J-2} \sum_{n} \frac{d(n)}{n^{J / 2}}|S(0, p-n ; q)| \sum_{l \mid p-n} l^{J-1} \sum_{c \leq \frac{2 \sqrt{X}}{q l}} n^{1 / \kappa} c^{J-2} \\
& \ll q^{-1+\varepsilon} \sum_{n} \frac{d(n) d(p-n)}{n^{J / 2-(J-1) / \kappa}}|S(0, p-n ; q)| .
\end{aligned}
$$

To this end, we choose $\kappa=2+\varepsilon / 2$ and $J$ large enough so that $J / 2-(J-1) / \kappa>1$. We hence obtain $Y_{1} \ll p^{1 / 2} q^{-1+\varepsilon}$ and, since the sum over $n$ in (9) converges, $Y_{2} \ll q^{-1+\varepsilon}$.

For $X>q$, similarly to (5), using the bound $x^{j} W^{(j)}(x) \ll_{j} x^{-2}$, we have

$$
x^{j} f^{(j)}(x) \ll_{j}(1+\sqrt{p} x)^{j} q^{-2}(c x)^{-4} .
$$

Lemma 7 then gives

$$
y(n) \ll c q \rho\left(\frac{1+\sqrt{p} \rho}{1+\sqrt{n} \rho}\right)^{J} \frac{q^{2}}{X^{2}} .
$$

For the range $n \leq \rho^{-\kappa}$, a better bound than (7) in this case is

$$
y(n) \ll \frac{\sqrt{p} X}{c q}(\log q) \frac{q^{2}}{X^{2}} .
$$

Since $q^{2} / X^{2} \ll 1$, all the previous estimates remain valid. The ony place where this is not the case is the sum over $n \ll\left(q^{2} / 2 \sqrt{X}\right)^{\kappa}$ in (8) . However, this sum is void for $X>q^{4} / 4$ and the former estimate still works in the larger interval $X \leq q^{4} / 4$. Also, the 
quantity saved $q^{2} / X^{2}$ is sufficient to allow the sum over the dyadic values of $X$ involved to converge. The lemma follows.

The proof of the theorem is complete.

\section{REFERENCES}

[1] J. B. Conrey, The mean-square of Dirichlet L-functions, http://arxiv.org/abs/0708.2699

[2] P. Deligne, Le conjecture de Weil I, Publ. Math. I.H.E.S. 43 (1974), 273-307

[3] H. Iwaniec, P. Sarnak, The non-vanishing of central values of automorphic L-functions and LandauSiegel zeros, Israel J. Math. 120 (2000), 155-177

[4] M. Jutila, Lectures on a method in the theory of exponential sums, Tata Lectures on Mathematics and Physics 80, Springer-Verlag, 1987

[5] E. Kowalski, P. Michel, A lower bound for the rank of $J_{0}(q)$, Acta Arith. 94 (2000), no. 4, 303-343

[6] E. Kowalski, P. Michel, J. M. VanderKam, Mollification of the fourth moment of automorphic L-functions and arithmetic applications, Invent. Math. 142 (2000), 95-151

[7] M. P. Young, The reciprocity law for the twisted second moment of Dirichlet L-functions, http://arxiv.org/abs/0708.2928

Mathematical Institute, University of Oxford, OXFORD, OX1 3LB

E-mail address: hung.bui@maths.ox.ac.uk 